\title{
Simple and Clean Photoinduced Aromatic Trifluoromethylation Reaction
}

\author{
$\mathrm{Lu} \mathrm{Li}^{\dagger,+}$ Xiaoyue $\mathrm{Mu},{ }^{\dagger}$ Wenbo Liu, ${ }^{\dagger}$ Yichen Wang, ${ }^{\ddagger}$ Zetian $\mathrm{Mi}^{*}, \stackrel{+}{\ddagger}$ and Chao-Jun $\mathrm{Li}^{*}{ }^{\dagger}$ \\ ${ }^{\dagger}$ Department of Chemistry, McGill University, 801 Sherbrooke Street West, Montreal, Quebec H3A 0B8, Canada \\ ${ }^{\ddagger}$ Department of Electrical and Computer Engineering, McGill University, 3480 University Street, Montreal, Quebec H3A 0E9, \\ Canada
}

Supporting Information

ABSTRACT: We describe a simple, metal- and oxidantfree photochemical strategy for the direct trifluoromethylation of unactivated arenes and heteroarenes under either ultraviolet or visible light irradiation. We demonstrated that photoexcited aliphatic ketones, such as acetone and diacetyl, can be used as promising low-cost radical initiators to generate $\mathrm{CF}_{3}$ radicals from sodium triflinate efficiently. The broad utility of this strategy and its benefit to medicinal chemistry are demonstrated by the direct trifluoromethylation of unprotected bidentate chelating ligand, xanthine alkaloids, nucleosides, and related antiviral drug molecules.

$\mathrm{T}$ he incorporation of trifluoromethyl $\left(\mathrm{CF}_{3}\right)$ group into drug candidates is widely prevalent in biochemical and medicinal science because the $\mathrm{CF}_{3}$ moiety can dramatically modify the physical and biological properties of parent molecules such as solubility, lipophilicity, and catabolic stability. ${ }^{1-4}$ During the past decades, an increasing attention has been paid to the trifluoromethylation of arenes and heteroarenes, which are fundamental building blocks of many top-selling pharmaceuticals and agrochemicals, including celecoxib, cinacalcet, nilotinib, beflubutamid, norfluazon, and so on. ${ }^{5}$ However, the traditional cross-coupling methodologies usually require stoichiometric amounts of metal complexes, preactivated substrates, or directing groups. ${ }^{6}$ Substrate scope also suffers from severe limitations due to the harsh reaction conditions. ${ }^{7}$ More recently, many powerful strategies have been developed to synthesize functionalized benzotrifluorides with high yield and broad substrate scope. ${ }^{8-10}$ One of the major improvements has been made via copper- or palladium-catalyzed coupling of various prefunctionalized aryl halides, boronic acids, and (hetero)arenes with either nucleophilic or electrophilic $\mathrm{CF}_{3}$ reagents. ${ }^{11-17}$ Pivotal progress was made by Langois, Baran, and MacMillan who pioneered the direct radical trifluoromethylation of arenes and heteroarenes. ${ }^{18-26}$ The prefunctionalized strategy usually results in a high regioselectivity, while the direct trifluoromethylation of unactivated arenes can avoid the multistep synthesis of complicated prefunctionalized substrates.

Specific to the direct radical trifluoromethylation strategy, the significant breakthrough made by MacMillan et al. ${ }^{19}$ showed that ruthenium and iridium photoredox catalysts are amenable to the direct trifluoromethylation of arenes under mild visible-light irradiation. In the meantime, Baran et al. ${ }^{20}$ described a powerful transition metal-free method for the direct trifluoromethylation of heterocycles by using sodium triflinate $\left(\mathrm{CF}_{3} \mathrm{SO}_{2} \mathrm{Na}\right.$, Langlois' reagent) and t-butyl hydroperoxide. However, the difficulty associated with the removal of metal catalyst residue has led to growing concerns in pharmaceuticals and materials. ${ }^{27}$ On the other hand, the preparation of concentrated peroxides, commonly used oxidants for the generation of $\mathrm{CF}_{3}$ radicals, is energy intensive and dangerous. In this respect, the development of a transition-metal-free trifluoromethylation methodology to access benzotrifluorides under safe and environmentally benign conditions has become an essential yet challenging objective for today's medical science.

As a greener alternative to the metal-catalyzed strategy in organic synthesis, photochemical reactions possess the advantage of avoiding the use of expensive and toxic metal catalysts. ${ }^{28-30}$ Very recently, we reported photoinduced metal-free aromatic Finkelstein $^{31}$ and Sonogashira ${ }^{32}$ reactions at room temperature. With particular interest in photochemistry and fluorine chemistry, we describe herein a mild, photoinduced approach for the direct trifluoromethylation of unactivated arenes and heteroarenes through a photoreduction mechanism without using any metal catalysts or oxidants. As shown in Scheme 1, this radical trifluoromethylation protocol was carried out in an exceptionally facile manner by simply mixing substrate and solid

Scheme 1. Approaches to the Generation of $\mathrm{CF}_{3}$ Radicals from Sodium Triflinate

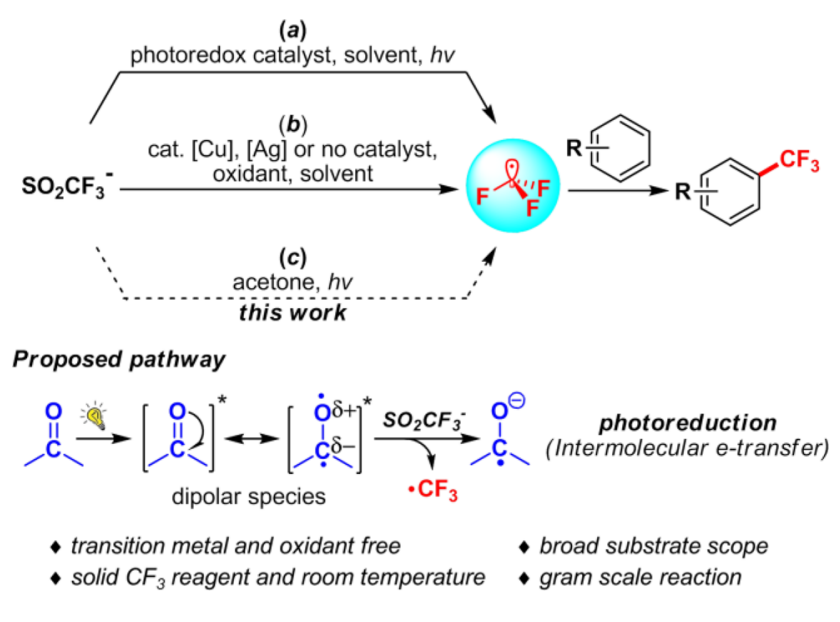

Received: March 16, 2016

Published: May 3, 2016 
sodium triflinate $\left(\mathrm{CF}_{3} \mathrm{SO}_{2} \mathrm{Na} \text {, Langlois' reagent }\right)^{18}$ in acetone (solvent) under UV light $(>300 \mathrm{~nm})$ irradiation. Moreover, we demonstrated that visible light $(>400 \mathrm{~nm})$ also worked smoothly if a small amount of diacetyl cosolvent was added.

As one of the most important and commonly used solvents in organic synthesis and chemical industry, acetone is also the simplest ketone and was among the first organic chromophoric compounds described in photoreactions. ${ }^{33}$ Numerous photoinduced processes have been successfully initiated by using $n, \pi^{*}$ triplet-excited ketones, such as hydrogen abstraction, ${ }^{34}$ oxetane formation (Paternò-Büchi reaction), ${ }^{35}$ photocyclization, ${ }^{36}$ and the Norrish reactions. ${ }^{37}$ As depicted in Figure 1a, acetone has a
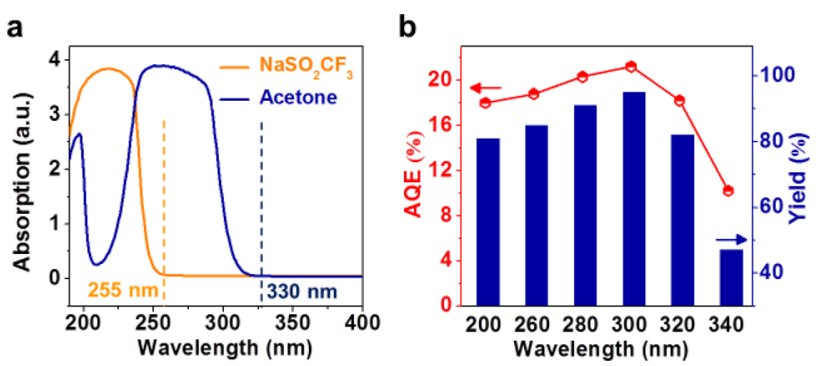

Figure 1. (a) $\mathrm{UV}$-vis absorption spectra of $\mathrm{CF}_{3} \mathrm{SO}_{2} \mathrm{Na}$ (orange line) and acetone (blue line). (b) Apparent quantum efficiency (AQE) (red line and hexagon symbol, left axis) and yield of 1 (blue columns, right axis) as a function of wavelength of the incident light.

strong absorption band in the ultraviolet range below $330 \mathrm{~nm}$. Its electronic excitation involves the transition of a lone pair electron from oxygen to the carbonyl carbon. Based on this "radical-like" characteristic, we envisioned that the resulting electron-deficient oxygen atom of the carbonyl $\mathrm{n}, \pi^{*}$ state may abstract one electron from $\mathrm{CF}_{3} \mathrm{SO}_{2} \mathrm{Na}$ to generate a $\mathrm{CF}_{3}$ radical, which is called the photoreduction of ketone. ${ }^{34}$

Initial experiments started with 1,3,5-trimethoxybenzene and sodium triflinate salts in acetone at $20^{\circ} \mathrm{C}$ under UV irradiation $(\lambda$ $=254 \mathrm{~nm}$ ) at an intensity of $4.0 \mathrm{~mW} \mathrm{~cm}^{-2}$ by using a photoreactor (Figure S1). The airtight quartz tube containing reactants and solvent was evacuated by four freeze-pump-thaw cycles and backfilled with ultrapurified argon (>99.999\%) prior to use. Optimized reaction conditions, which include the use of $0.1 \mathrm{mmol}$ of substrate and 4 equiv of triflinate in $1 \mathrm{~mL}$ of acetone, gave the desired $\mathrm{CF}_{3}$-substituted products 1 in excellent yield of $88 \%$ after $15 \mathrm{~h}$ photoreaction. Obviously, the conversion rate and yield of a photochemical reaction depends strongly on the incident light intensity. Therefore, if a $300 \mathrm{~W}$ xenon lamp with a stronger light intensity of $20 \mathrm{~mW} \mathrm{~cm}^{-2}$ around $250 \mathrm{~nm}$ was employed (Figure S1), the reaction time could be dramatically reduced from 15 to $2 \mathrm{~h}$ with a good yield of $81 \%$. It is noted that $\sim 5 \%$ of bis- $\mathrm{CF}_{3}$-substituted product 2 was also detected after $2 \mathrm{~h}$ irradiation with strong UV light at $20 \mathrm{~mW} \mathrm{~cm}^{-2}$. Further extending the reaction time to 4 and $20 \mathrm{~h}$ increased the yield of bis- $\mathrm{CF}_{3}$-substituted product 2 to $36 \%$ and $77 \%$, respectively. Control experiments established the requirement of UV light, as no reaction proceeded in the dark even under heating.

To demonstrate the effect of acetone on the photoinduced trifluoromethylation process, a series of optical filters and different solvents were employed to run this reaction under UV irradiation from the $300 \mathrm{~W}$ xenon lamp (emission wavelengths between 200 and $1000 \mathrm{~nm}$ ). As shown in Figure 1a, sodium triflinate itself has a wide absorption band below $255 \mathrm{~nm}$ in the $\mathrm{UV}$-vis spectrum, indicating that UV light shorter than $255 \mathrm{~nm}$ may directly activate sodium triflinate salts. Experimental results showed that under direct photoirradiation from a $300 \mathrm{~W}$ xenon lamp without any filter, a substantial yield of $\mathbf{1}$ could also be achieved by using either acetonitrile $(53 \%)$ or dichloromethane $(66 \%)$ instead of acetone as solvent (Table S1). However, if a $280 \mathrm{~nm}$ long-pass filter was carefully mounted in the system to completely block wavelengths shorter than $280 \mathrm{~nm}$ from the xenon lamp, which may interact with sodium triflinate, the reaction did not proceed at all in acetonitrile or dichloromethane; whereas $83 \%$ yield of 1 could be obtained in acetone. These observations clearly demonstrate that photoexcited acetone is capable of triggering the trifluoromethylation reaction efficiently. Therefore, acetone in this study essentially acts as both solvent to dissolve reactants and photosensitizer to harvest resonant photons. $^{38,39}$

The optimal wavelength and effective range for the trifluoromethylation reaction in acetone were determined by the wavelength dependence experiment. As shown in Figure 1b, the best yield of $95 \%$ ( $0.1 \mathrm{mmol}$ scale $)$ for 1 could be achieved under $2 \mathrm{~h}$ photoirradiation from a $300 \mathrm{~W}$ xenon lamp with a 300 $\mathrm{nm}$ long-pass filter $(\lambda>300 \mathrm{~nm})$. The apparent quantum efficiency at $300 \mathrm{~nm}$ was calculated to be $\sim 22 \%$ (the calculation details can be found in the SI). The yield of 1 still remained at a high level around $320 \mathrm{~nm}$ but decreased dramatically when the wavelength of light irradiation was longer than $340 \mathrm{~nm}$. The effective wavelength range is consistent with the absorption spectrum of acetone, further indicating that acetone was a good photosensitizer for the trifluoromethylation reaction. A proposed mechanism is depicted in Figure 2.

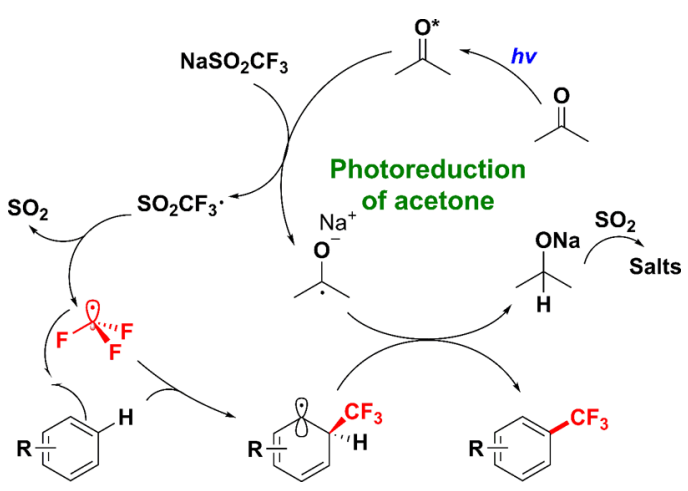

Figure 2. Proposed mechanism.

The preparative scope of the trifluoromethylation reaction was carried out under UV irradiation with either a $300 \mathrm{~W}$ xenon lamp $(\lambda>300 \mathrm{~nm})$ or the photoreactor $(\lambda=254 \mathrm{~nm})$. In general, this clean photochemical protocol allows the direct incorporation of $\mathrm{CF}_{3}$ moiety into a broad range of arenes, heteroarenes, and nucleosides in yields ranging from $38 \%$ to $95 \%$ (Scheme 2). Evidently, this approach is very effective for electron-rich arenes $(1,2,12)$ since $\mathrm{CF}_{3}$ radical is electron deficient. ${ }^{40}$ In addition, although we cannot explain the phenomenon in detail yet, it was found that the addition of $60 \mu \mathrm{L}$ of acetic anhydride (Figure S2) into acetone could increase the trifluoromethylation yield (Table S2). Therefore, unsubstituted benzene and other arenes with either electron-withdrawing groups or weak electron-donating groups gave good yields $(3-5,9,13)$ by adding $60 \mu \mathrm{L}$ of acetic anhydride as additive. For unsymmetrical aromatics with more than one reactive site, products may be formed as isomeric mixtures $(6-8,14,15)$ or a single compound (16), synergistically governed by the innate electronic effect and steric effect of 
Scheme 2. Radical Trifluoromethylation of Various Arenes and Heteroarenes ${ }^{a, b}$

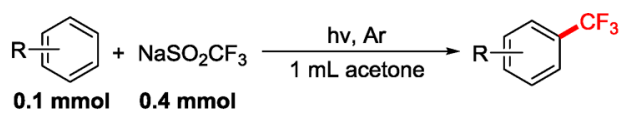

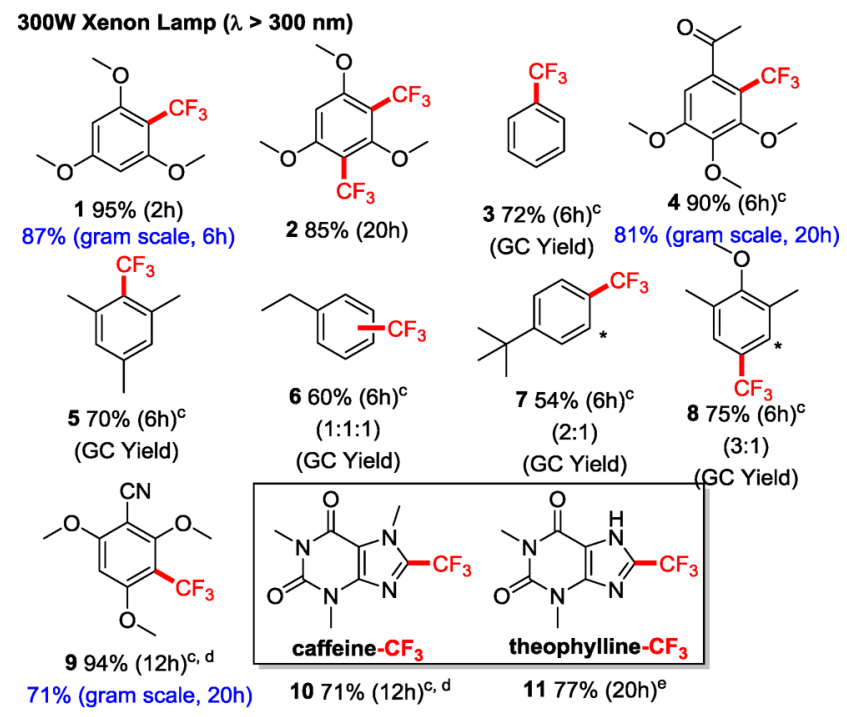

Photoreactor $(\lambda=254 \mathrm{~nm})$<smiles>COc1cc(C)c(C(F)(F)F)c(OC)c1OC</smiles>

$1275 \%$ (40h)<smiles>CC(C)(C)c1cc(C(F)(F)F)cc(C(C)(C)C)c1O</smiles>

$1649 \%(40 h)^{c}$<smiles>CC(=O)c1c(C)cc(C)c(C(F)(F)F)c1C</smiles>

$1376 \%(40 h)^{c, d}$<smiles>COc1ccc(C(F)(F)F)c(OC)n1</smiles><smiles>COc1cc(C)c(C(F)(F)F)cc1OC</smiles>

$1477 \%(40 h)^{\mathrm{c}}$

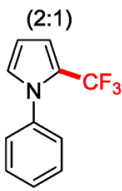

$1764 \%(40 h)^{c}$<smiles>COc1ccc(C(F)F)c(OC)c1</smiles>

$1567 \%(40 h)^{d}$

$(2: 1)$

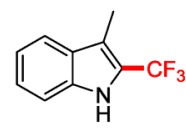

$1972 \%(40 \mathrm{~h})$

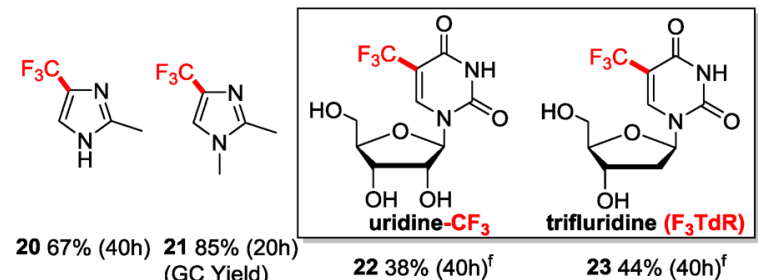

${ }^{a}$ Reaction conditions: arenes and heteroarenes $(0.1 \mathrm{mmol})$, $\mathrm{NaSO}_{2} \mathrm{CF}_{3}(0.4 \mathrm{mmol})$, and acetone $(1.0 \mathrm{~mL})$ at $20{ }^{\circ} \mathrm{C}$ in argon under UV light for $2-40 \mathrm{~h}$. ${ }^{b}$ Yield of isolated product. ${ }^{c} 0.06 \mathrm{~mL}$ of acetic anhydride was added into $0.94 \mathrm{~mL}$ of acetone. ${ }^{d}$ Reaction showed incomplete conversion, and a second portion of fresh $\mathrm{NaSO}_{2} \mathrm{CF}_{3}(0.4 \mathrm{mmol})$ was added into the system to drive the reaction toward completion. ${ }^{e} 0.05 \mathrm{~mL}$ of water was added into 0.95 $\mathrm{mL}$ of acetone. ${ }^{f} 0.1 \mathrm{~mL}$ of water was added into $0.9 \mathrm{~mL}$ of acetone. *Minor isomeric product.

the substrates. Besides arenes, a variety of heteroarenes including pyridine (17), pyrrole (18), indole (19), and imidazoles $(20,21)$ could all be likewise tolerated with this photochemical procedure. To further demonstrate the broad utility of this strategy, some biologically active molecules, such as caffeine (10), theophylline (11), and uridine (22), were successfully subjected to this simple protocol to access their trifluoromethylated derivatives with high regioselectivity. In these cases, the

addition of a small amount of water $(0.05$ or $0.1 \mathrm{~mL})$ into acetone could improve the substrate solubility and get a better yield. Moreover, 5-trifluoromethyl-2'-deoxyuridine (trifluridine, $\mathrm{F}_{3} \mathrm{TdR}, 23$ ), a powerful antiviral drug that has been shown to be highly useful in many biological systems with wide clinical utility, ${ }^{41}$ was synthesized in a moderate yield via the direct trifluoromethylation of $2^{\prime}$-deoxyuridine without protection. Moreover, we conducted a large-scale reaction in a $100 \mathrm{~mL}$ of quartz flask, and the results revealed that our strategy was effective on the gram scale $(1,4$, and $9,5 \mathrm{mmol})$. Unfortunately, substrates with nitro and carboxyl groups did not give good yields. Amino group is not tolerated in our system due to its intrinsic reactivity with acetone, generating imines.

Encouraged by the experimental outcome under UV light, we attempted to further extend this strategy into visible region $(\lambda>$ $400 \mathrm{~nm}$ ) by employing aliphatic diacetyl instead of acetone as the radical initiator, which contains two carbonyl groups and shows absorption in the visible wavelength range (Scheme 3a). As shown in Scheme 3b, the preliminary results demonstrated that a

Scheme 3. (a) UV-vis Absorption Spectrum of Diacetyl and Reaction Setup (inset), (b) Control Experiments with or without Diacetyl under Visible Light Irradiation, and (c) Substrate Scope under Visible Light Irradiation

a

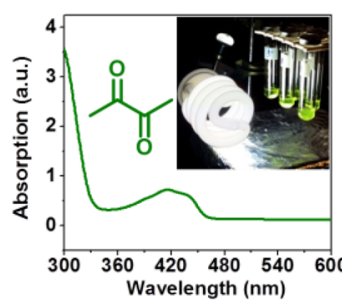

C

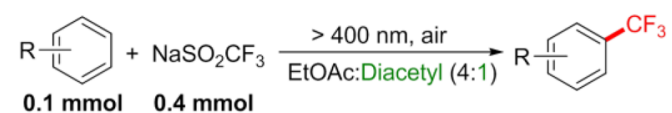

300W Xenon Lamp $(\lambda>400 \mathrm{~nm})^{\mathrm{a}, \mathrm{b}}$<smiles>COc1cc(OC)c(C(F)(F)F)c(OC)c1</smiles>

$193 \%(10 h) \quad 491 \%(40 h) \quad 989 \%(40 h) \quad 1082 \%(20 h)$<smiles>CC(C)(C)c1cc(C(C)(C)C)c(O)c(C(C)(C)C)c1</smiles><smiles>Cc1c(C(F)(F)F)[nH]c2ccccc12</smiles><smiles>COc1ccc(OC)c(C(F)(F)F)c1</smiles>

$1665 \%(20 h)$

$1975 \%(40 h)$ $2441 \%(10 h)$<smiles>COc1cc(C=O)c(C(F)(F)F)c(OC)c1OC</smiles><smiles>CC(C)c1cc(C(F)(F)F)cc(C(C)C)c1Br</smiles><smiles>Cc1cc(I)c(I)c(C)c1Cl</smiles><smiles>FC(F)(F)c1nc2ncncc2[nH]1</smiles><smiles>COc1ccnc(-c2cc(OC)c(C(F)(F)F)cn2)c1</smiles>

$2655 \%(40 h)$

$2751 \%(40 h) \quad 2865 \%(20 h)^{c}$

$2942 \%(20 h)$

${ }^{a}$ Reaction conditions: arenes and heteroarenes $(0.1 \mathrm{mmol})$, $\mathrm{NaSO}_{2} \mathrm{CF}_{3}(0.4 \mathrm{mmol})$ and a mixture of ethyl acetate $(0.8 \mathrm{~mL})$ diacetyl $(0.2 \mathrm{~mL})$ at $20^{\circ} \mathrm{C}$ in Ar under visible light for $10-40$ h. ${ }^{b}$ Yield of isolated product. ${ }^{c}$ Water was used instead of ethyl acetate as solvent. 
good yield of $66 \%$ for the trifluoromethylation of mesitylene could be achieved under visible light irradiation with a household fluorescent light bulb in a mixture solution of acetone-diacetyl (9:1). Besides acetone, a variety of other solvents, such as acetonitrile, ethyl acetate, and water, also worked well with diacetyl (Table S3). In contrast, no reaction occurred in pure solvent without diacetyl under the same conditions, indicating the crucial role of diacetyl for the visible light-induced trifluoromethylation reaction. Finally, the reaction scope was tested under the optimized reaction conditions, which include the use of 4 equiv of triflinate in $1.0 \mathrm{~mL}$ of ethyl acetate-diacetyl mixture (4:1) under visible light irradiation from a $300 \mathrm{~W}$ xenon lamp with a $400 \mathrm{~nm}$ long-pass filter (Scheme 3c). It was found that most substrates listed in Scheme 2 proceeded well in the visible light system with comparable yields. Importantly, the reaction was also compatible with aldehyde (25) and halogen $(26,27)$ groups, which are not stable under UV irradiation. Purine (28) and bipyridine (29), widely used nitrogencontaining heterocycles and bidentate chelating ligands, respectively, were also operative smoothly with high regioselectivity by this method.

In summary, an efficient and practical approach to the photoinduced trifluoromethylation of arenes and heteroarenes was developed with easily handled sodium triflinate. The value of this strategy has been highlighted via the trifluoromethylation of biologically active molecules under either UV or visible light irradiation. Significantly, this photochemical strategy employed acetone, one of the most widely used and cheapest organic solvents, instead of expensive metal catalyst or dangerous peroxides to generate $\mathrm{CF}_{3}$ radical, which provides a greener route to cost-effective large-scale synthesis of trifluoromethylated chemicals.

\section{ASSOCIATED CONTENT}

\section{S Supporting Information}

The Supporting Information is available free of charge on the ACS Publications website at DOI: 10.1021/jacs.6b02782.

Experimental details and data (PDF)

\section{AUTHOR INFORMATION}

\section{Corresponding Authors}

*zetian.mi@mcgill.ca

*cj.li@mcgill.ca

\section{Notes}

The authors declare no competing financial interest.

\section{ACKNOWLEDGMENTS}

This work was financially supported by the Canada Research Chair (Tier 1) foundation, the Natural Sciences and Engineering Research Council of Canada, the Fonds de recherché sur la nature et les technologies, Canada Foundation for Innovation (CFI), and McGill University.

\section{REFERENCES}

(1) Müller, K.; Faeh, C.; Diederich, F. Science 2007, 317, 1881.

(2) Hagmann, W. K. J. Med. Chem. 2008, 51, 4359.

(3) Meanwell, N. A. J. Med. Chem. 2011, 54, 2529.

(4) Zhou, Y.; Wang, J.; Gu, Z.; Wang, S.; Zhu, W.; Aceña, J. L.; Soloshonok, V. A.; Izawa, K.; Liu, H. Chem. Rev. 2016, 116, 422.

(5) Alonso, C.; Martínez de Marigorta, E.; Rubiales, G.; Palacios, F. Chem. Rev. 2015, 115, 1847.

(6) Tomashenko, O. A.; Grushin, V. V. Chem. Rev. 2011, 111, 4475.
(7) Burton, D. J.; Yang, Z.-Y. Tetrahedron 1992, 48, 189.

(8) Furuya, T.; Kamlet, A. S.; Ritter, T. Nature 2011, 473, 470.

(9) Chen, M.; Buchwald, S. L. Angew. Chem., Int. Ed. 2013, 52, 11628.

(10) Beatty, J. W.; Douglas, J. J.; Cole, K. P.; Stephenson, C. R. J. Nat. Commun. 2015, 6, 7919.

(11) Cho, E. J.; Senecal, T. D.; Kinzel, T.; Zhang, Y.; Watson, D. A.; Buchwald, S. L. Science 2010, 328, 1679.

(12) Wang, X.; Truesdale, L.; Yu, J.-Q. J. Am. Chem. Soc. 2010, 132, 3648.

(13) Morimoto, H.; Tsubogo, T.; Litvinas, N. D.; Hartwig, J. F. Angew. Chem., Int. Ed. 2011, 50, 3793.

(14) Liu, T.; Shen, Q. Org. Lett. 2011, 13, 2342.

(15) Senecal, T. D.; Parsons, A. T.; Buchwald, S. L. J. Org. Chem. 2011, $76,1174$.

(16) Chu, L.; Qing, F.-L. J. Am. Chem. Soc. 2012, 134, 1298.

(17) Besset, T.; Schneider, C.; Cahard, D. Angew. Chem., Int. Ed. 2012, $51,5048$.

(18) Langlois, B. R.; Laurent, E.; Roidot, N. Tetrahedron Lett. 1991, 32, 7525.

(19) Nagib, D. A.; MacMillan, D. W. C. Nature 2011, 480, 224.

(20) Ji, Y.; Brueckl, T.; Baxter, R. D.; Fujiwara, Y.; Seiple, I. B.; Su, S.; Blackmond, D. G.; Baran, P. S. Proc. Natl. Acad. Sci. U. S. A. 2011, 108, 14411.

(21) Ye, Y.; Lee, S. H.; Sanford, M. S. Org. Lett. 2011, 13, 5464.

(22) Ye, Y.; Künzi, S. A.; Sanford, M. S. Org. Lett. 2012, 14, 4979.

(23) Cui, L.; Matusaki, Y.; Tada, N.; Miura, T.; Uno, B.; Itoh, A. Adv. Synth. Catal. 2013, 355, 2203.

(24) Sladojevich, F.; McNeill, E.; Börgel, J.; Zheng, S.-L.; Ritter, T. Angew. Chem., Int. Ed. 2015, 54, 3712.

(25) Natte, K.; Jagadeesh, R. V.; He, L.; Rabeah, J.; Chen, J.; Taeschler, C.; Ellinger, S.; Zaragoza, F.; Neumann, H.; Brückner, A.; Beller, M. Angew. Chem., Int. Ed. 2016, 55, 2782.

(26) Lefebvre, Q.; Hoffmann, N.; Rueping, M. Chem. Commun. 2016, 52, 2493.

(27) Sun, C.-L.; Shi, Z.-J. Chem. Rev. 2014, 114, 9219.

(28) Li, L.; Fan, S.; Mu, X.; Mi, Z.; Li, C.-J. J. Am. Chem. Soc. 2014, 136, 7793.

(29) Sun, J.; Peng, X.; Guo, H. Tetrahedron Lett. 2015, 56, 797.

(30) Mfuh, A. M.; Doyle, J. D.; Chhetri, B.; Arman, H. D.; Larionov, O. V. J. Am. Chem. Soc. 2016, 138, 2985.

(31) Li, L.; Liu, W.; Zeng, H.; Mu, X.; Cosa, G.; Mi, Z.; Li, C.-J. J. Am. Chem. Soc. 2015, 137, 8328.

(32) Liu, W.; Li, L.; Li, C.-J. Nat. Commun. 2015, 6, 6526.

(33) Coyle, J. D.; Carless, H. A. J. Chem. Soc. Rev. 1972, 1, 465.

(34) Singh, P. J. Chem. Soc. C 1971, 714.

(35) Büchi, G.; Inman, C. G.; Lipinsky, E. S. J. Am. Chem. Soc. 1954, 76, 4327.

(36) Yang, N. C.; Yang, D.-D. H. J. Am. Chem. Soc. 1958, 80, 2913.

(37) Chatgilialoglu, C.; Crich, D.; Komatsu, M.; Ryu, I. Chem. Rev. 1999, 99, 1991.

(38) Xia, J.-B.; Zhu, C.; Chen, C. J. Am. Chem. Soc. 2013, 135, 17494

(39) Xia, J.-B.; Zhu, C.; Chen, C. Chem. Commun. 2014, 50, 11701.

(40) Dolbier, W. R. Chem. Rev. 1996, 96, 1557.

(41) Heidelberger, C. Cancer Res. 1970, 30, 1549. 